\title{
Factores de riesgo y tiempo de hipotermia neonatal en una terapia intensiva en Tamaulipas
}

\author{
Patricia Rivera-Vázquez ${ }^{1 *}$, Guadalupe L. Rodríguez-Tovar ${ }^{1,2}$ y Oscar M. Berlanga-Bolado \\ ${ }^{1}$ Unidad de Cuidados Intensivos Neonatales Hospital General de Ciudad Victoria "Dr. Norberto Treviño Zapata"; ${ }^{2}$ División de Pediatría Hospital \\ Regional de Alta Especialidad de Ciudad Victoria "Bicentenario 2010". Ciudad Victoria, Tamaulipas, México
}

\section{Resumen}

Antecedentes: La hipotermia afecta la función fisiológica en el neonato ligada a la sobrevida y a la morbilidad asociada, por lo que los factores de riesgo deben ser atendidos tempranamente por el equipo de salud en el ámbito hospitalario. Objetivo: Determinar la asociación entre factores de riesgo y el tiempo de hipotermia del neonato en una unidad de cuidados intensivos en Tamaulipas. Material y métodos: Estudio retrospectivo, descriptivo correlacional en 155 neonatos seleccionados a través de muestreo probabilístico aleatorio simple. Datos recabados en formato ex profeso, analizados con programa SPSS V.23. mediante análisis bivariado utilizando odds ratio y Ji cuadrado de Pearson. Resultados: Presentaron hipotermia moderada 142 (91.6\%) casos, el tiempo de hipotermia trascurrido fue en promedio de 14 horas. Los factores de riesgo asociados fueron la edad ( $\left.X^{2}: 591.71 ; p<0.05\right)$, presencia de anomalías congénitas (OR: 1.005; IC 95\%: 0.885-1.141, $>6$ horas [OR: 1.232; IC 95\%: 0.927-1.636]), retardo en el crecimiento intrauterino (RCIU) (OR: 1.070; IC 95\%: 0.981-1.167, $>6$ horas [OR: 1.534; IC 95\%: 1.217-1.934]), obtenidos por cesárea (OR: 1.009; IC 95\%: 0.892-1.141, < 6 horas [OR: 1.215; IC 95\%: 0.759-1.944]), contacto con objetos no precalentados (OR: 1.421; IC 95\%: 0.458-4.410, < 6 horas [OR: 1.396; IC 95\%: 0.904-2.157]) y contacto con circulación de aire frío (OR: 1.156; IC 95\%: 0.396-3.375, < 6 horas [OR: 1.280; IC 95\%: 0.8461.937]). Conclusión: La identificación de factores de riesgo en nuestro entorno pone de manifiesto la relevancia de la valoración y detección temprana que permitan establecer planes de cuidado seguros y de calidad acordes a las necesidades del neonato.

Palabras clave: Riesgo. Tiempo. Hipotermia neonatal. Enfermería.

\section{Risk factors and time of neonatal hypothermia in an intensive therapy in Tamaulipas}

\section{Abstract}

Background: Hypothermia affects the physiological function in the newborn linked to survival and the associated morbidity, so the risk factors must be addressed early by the health team in the hospital setting. Objective: To determine the association between risk factors and the hypothermia time of the newborn in an intensive care unit in Tamaulipas. Material and methods: Retrospective, descriptive-correlational study in 155 neonates selected through simple random probabilistic sampling. Data collected in an express format, analyzed with the SPSS V.23 program. Using bivariate analysis using odds ratio (OR) and Chi-square of Pearson. Results: They presented moderate hypothermia 142 (91.6\%), the hypothermia

Correspondencia:

*Patricia Rivera-Vázquez

E-mail: pattyri@live.com.mx
Disponible en internet: 16-12-2019 Perinatol Reprod Hum. 2019;33:47-57 www.perinatologia.mx
Fecha de recepción: 09-01-2019 Fecha de aceptación: 07-11-2019 DOI: 10.24875/PER.19000001 licencia CC BY-NC-ND (http://creativecommons.org/licenses/by-nc-nd/4.0/). 
Perinatol Reprod Hum. 2019;33

time elapsed was on average $14 \mathrm{~h}$, the associated risk factors were age $(\mathrm{X} 2=591.71 ; p<0.05)$, presence of congenital anomalies (OR: 1.005; 95\% confidence intervals [Cl]: 0.885-1.141, > $6 \mathrm{~h}$ [OR: 1.232; 95\% Cl: 0.927-1.636]), delay in intrauterine growth (OR: 1.070; 95\% Cl: 0.981-1.167, > 6 h [OR: 1.534; 95\% Cl: 1.217-1.934), obtained by caesarean section (OR: 1009; Cl 95\%: 0.892-1.141, < 6 h [OR: 1.215; Cl 95\%: 0.759-1.944]). Contact with non-preheated objects (OR: 1.421; 95\% Cl: 0.458-4.410, < 6 h [OR: 1.396; 95\% Cl: 0.904-2.157]), contact with cold air circulation (OR: 1.156; 95\% Cl: 0.396-3.375, < 6 h [OR: 1.280; 95\% Cl: 0.846-1.937]). Conclusion: The identification of risk factors in our environment highlights the relevance of the assessment and early detection to establish safe and quality care plans according to the needs of the newborn.

Key words: Risk. Time. Neonatal hypothermia. Nursing.

\section{Antecedentes}

La North American Nursing Diagnosis Association ${ }^{1}$ define la hipotermia como la temperatura por debajo del rango normal, que en el recién nacido es de 36.5 a $37.5^{\circ} \mathrm{C}$. Hoy en día, a pesar de la importancia que reviste la temperatura en el proceso de adaptación del recién nacido como una variable fisiológica y que se refleja como signo vital del recién nacido, se ha observado que la temperatura corporal en el neonato ha sido poco estudiada como tal y, por lo tanto, es poco documentada y reconocida, y, por consiguiente, mal manejada. En este sentido, los estudios realizados al respecto demuestran que la hipotermia neonatal por sí sola aumenta la mortalidad y la morbilidad, ya que afecta la función fisiológica en el neonato. Esto va ligado a la sobrevida, al estado de salud y a la morbilidad asociada, por lo que se considera como un factor de riesgo que debe ser atendido por el equipo de salud en la atención del recién nacido desde su nacimiento en el ámbito hospitalario ${ }^{2,3}$, ya que se considera que, cuando se presenta hipotermia posterior al nacimiento, ello interfiere en el proceso de adaptación del neonato y retrasa una evolución clínica favorable debido a los posibles efectos metabólicos y hemodinámicos que en él ocurren, lo que contribuye a la presencia de complicaciones derivadas del resultado del metabolismo basal disminuido que presenta el neonato con hipotermia ${ }^{3}$.

Por lo tanto, la hipotermia se considera como un importante contribuyente a la morbilidad y mortalidad neonatal, especialmente en entornos de bajos recursos, ya que la mayoría de las embarazadas no cuenta con servicio médico que facilite a través del control prenatal la identificación de factores de riesgo y, con ello, una correcta planeación para la atención del parto y del recién nacido en las mejores condiciones ambientales y de salud que pueden ofrecer las instituciones encargadas para ello². Sin embargo, en estos se logra un progreso limitado hacia la comprensión del riesgo de mortalidad después de la hipotermia, que depende tanto del grado o la gravedad del tiempo de exposición a una temperatura no adecuada, así como de la identificación temprana de factores como la edad gestacional, el peso del recién nacido, sus antecedentes patológicos y no patológicos, características y problemas al nacer, así como las intervenciones realizadas por el equipo de salud para mitigar la exposición al riesgo asociado $^{4,5}$. Lo anterior derivado de la aplicación de medidas para lograr un control térmico efectivo en el neonato, que, según lo reportado en la literatura, se iniciaron a finales del siglo XIX por Pierre Budín quien, con el uso de la incubadora neonatal, reportó una disminución de la mortalidad del 66 al $38 \%$ en neonatos a los cuales se proporcionó medidas de control término que siguen vigentes en la actualidad, especialmente en pretérminos, en las unidades de cuidados intensivos. Hoy en día sigue siendo responsabilidad de todo el equipo de salud, especialmente de enfermería, que está presente los 365 días del año durante las 24 horas del día en la atención neonatal y es quien valora, planea, ejecuta y evalúa la eficacia de las intervenciones realizadas en el cuidado del recién nacido de riesgo ${ }^{5}$, y quien aplica medidas que faciliten una termorregulación eficaz con el uso correcto de dispositivos de apoyo térmico, como es la incubadora neonatal en el ámbito hospitalario, que es un equipo biomédico de gran relevancia en la atención del recién nacido, especialmente en el prematuro, para prevenir las pérdidas de calor innecesarias durante su atención en la unidad de cuidados intensivos neonatales (UCIN).

Al respecto, la Sociedad Iberoamericana de Neonatología (2010) define la termorregulación como la habilidad de mantener un equilibrio entre la producción y la pérdida de calor para que la temperatura corporal esté dentro del rango normal, y la clasifica de acuerdo a su gravedad en el recién nacido. En este sentido, define como hipotermia leve la temperatura corporal que se encuentra entre 36 y $36.4^{\circ} \mathrm{C}$, hipotermia moderada la temperatura en el rango entre 32 y $35.9{ }^{\circ} \mathrm{C}$, e hipotermia grave la temperatura del neonato que se encuentra por debajo de los $32{ }^{\circ} \mathrm{C}$. En el recién nacido, la capacidad de producir calor es limitada y los mecanismos de pérdida del 
mismo suelen estar aumentados según la edad gestacional y los cuidados brindados en el momento del nacimiento. Lo mismo durante el periodo de adaptación, especialmente en la primera hora de vida extrauterina, denominada como «la hora dorada del recién nacido» 6,7 , por lo que el personal de salud debe estar atento a los factores de riesgo que contribuyen a la presencia de hipotermia neonatal para minimizar su efecto nocivo en la salud y evolución clínica del recién nacido.

En nuestro país, la Norma Oficial Mexicana NOM007-SSA2-2016, para la atención de la mujer durante el embarazo, parto y puerperio y de la persona recién nacida, menciona que se debe vigilar y tomar signos vitales a la persona recién nacida por lo menos una vez por turno (cada 8 horas) y que se debe vigilar estrechamente por lo menos durante 24 horas a toda persona recién nacida que haya recibido maniobras de reanimación neonatal o a aquellos productos pretérmino o postérmino con factores de riesgo que puedan afectar su pronóstico de salud ${ }^{8}$.

En este sentido se ha descrito que, inmediatamente posterior al nacimiento, el recién nacido requiere de una serie de valoraciones, procedimientos y cuidados que faciliten el proceso de adaptación al medio extrauterino, para minimizar con ello las complicaciones derivadas de la hipotermia, entre las que se encuentran la identificación de riesgos para la salud y las medidas para favorecer la termorregulación eficaz, entre las cuales se considera el control térmico como el eje central de la atención del equipo de salud desde el área de labor hasta la UCIN de una manera sistemática, donde las acciones permiten la detección temprana y el tratamiento oportuno ${ }^{9}$.

Para ello es necesario renonocer que existen factores de riesgo asociados a la presencia de hipotermia neonatal, entre los que se encuentran ser producto pretérmino, que se asocia además con un mayor riesgo de muerte neonatal temprana y tardía, ya que se considera que a menor edad gestacional, menor tono muscular, menos depósitos de grasa parda y menor habilidad de generar calor, lo que produce una mayor pérdida de calor desde el interior del organismo del recién nacido, especialmente en las primeras horas de nacimiento ${ }^{2,10}$. Otro factor asociado es que el neonato sea hijo de madre con hipotermia, especialmente los nacidos por cesárea, debido al proceso de sedación que influye en la disminución de la actividad física y el tono muscular y, ante ello, el neonato no puede usar la posición en flexión para reducir el área de superficie expuesta, lo que hace que se presente una respuesta muscular involuntaria como es el escalofrío ${ }^{11}$.
El ser valorados con una puntuación de Apgar baja al nacer, así como contar con antecedentes de aplicación de maniobras de reanimación en sus distintas formas y ser neonatos con antecedentes de hipoglucemia, son factores de riesgo para hipotermia, ya que se considera que dichos factores interfieren en la producción de calor, especialmente en los pretérmino, a pesar de que estos actúan a la vez como factor y consecuencia cuando se utiliza el mecanismo metabólico para corregir la temperatura durante el periodo de adaptación neonatal, lo que propicia un circulo vicioso ${ }^{11,12}$.

El retardo en el crecimiento intrauterino (RCIU) es considerado como un factor de riesgo para la presencia de hipotermia debido a que propicia una respuesta seudomotora no desarrollada, tono muscular y actividad disminuidas en el neonato, de forma similar a lo que ocurre con el prematuro, donde a menor peso, menor crecimiento y desarrollo, que se refleja en un pobre tono muscular, menor habilidad para la generación de calor debido al pobre depósito de grasa parda $y$, con ello, un incremento de pérdida de calor del recién nacido. Asimismo, la presencia de anomalías congénitas que impliquen apertura en la piel, como la gastrosquisis, onfalocele, extrofia vesical, meningocele, son factores que se deben tomar en cuenta, ya que también favorecen la presencia de hipotermia neonatal debido a que se incrementa la pérdida de calor a través de los mecanismos de evaporación y conducción, así también, la presencia de trastornos que afecten el sistema nervioso central que puedan interferir con la capacidad termorreguladora del recién nacido $0^{6,13}$ y motivar con ello la presencia de hipotermia de difícil control para el personal de salud.

Existen, además, otros factores de riesgo que están relacionados con los procedimientos y manejo del recién nacido, que se encuentran presentes desde la sala de expulsión, en el momento del traslado del neonato y al ingreso al área de terapia intensiva, especialmente cuando no existe una correcta valoración y planeación de las actividades a realizar para la atención del neonato, como son la utilización de incubadoras y/o cunas térmicas sin la regulación térmica adecuada de acuerdo a las necesidades del recién nacido, el usar sábanas y ropa hospitalaria no precalentadas, evitar el contacto piel a piel y el apego inmediato para la lactancia materna al momento del nacimiento. Del mismo modo, el exponerlo al aire frío sin precaución, administrar líquidos intravenosos a temperatura ambiental y realizar el baño del neonato en las primeras horas de nacido producen pérdidas de calor a través de los diferentes 
mecanismos (conducción, radiación, convección y evaporación) que pueden ser prevenibles por el personal de enfermería, ya que son factores de riesgo que pueden ser modificables y, con ello, evitar la presencia de hipotermia en el neonato $0^{4,9,13-15}$.

Al respecto, cabe mencionar que la hipotermia se ha utilizado como medida terapéutica en neonatos con encefalopatía hipóxico-isquémica, ya que sus mecanismos de acción provocan reducción del metabolismo cerebral, lo que previene el edema, disminuye la utilización de la energía y suprime la acumulación de aminoácidos citotóxicos y óxido nítrico. Así, se inhibe la activación plaquetaria y la cascada inflamatoria, lo que suprime la actividad de los radicales libres e inhibe la apoptosis neuronal, lo que reduce la extensión de la lesión cerebral provocada en estos neonatos. Debe iniciarse antes de las 6 horas del nacimiento hasta que el neonato cumpla 72 horas $^{16}$, contrario a lo que se recomienda en neonatos con necesidad de termorregulación eficaz, donde la presencia de factores de riesgo hacen que el personal de salud involucrado se prepare con anticipación para evitar pérdidas de calor innecesarias que produzcan hipotermia y, con ello, deriven complicaciones asociadas ${ }^{17}$.

Vemos, por tanto, que la detección temprana del riesgo de hipotermia en el neonato es de vital relevancia para prevenir posibles complicaciones, ya que la hipotermia provoca efectos de vasoconstricción en brazos y piernas, lo que evita que la sangre llegue a la superficie de la piel donde se produce la pérdida de calor. Si hay una vasoconstricción prolongada, afecta a la perfusión y la oxigenación de los tejidos, lo que provoca incremento en el consumo de oxígeno, hipoxia tisular y acidosis, especialmente en el prematuro, en el que existe una inadecuada cantidad de grasa parda en mediastino, escápula, axila y riñones, donde el estrés térmico genera, además, un incremento en el consumo de glucosa, se libera ácido láctico y se produce hipoglucemia. Dado que la glucosa es la fuente primaria de energía para el cerebro, cuando esta se presenta, el nivel de conciencia del neonato disminuye, hay respiración lenta, lo que provoca dificultad respiratoria, falla aguda renal, coagulación diseminada y un incremento en la incidencia de infección y persistencia del conducto arterioso ${ }^{18}$.

Al respecto, la literatura menciona que, para evitar una vasoconstricción prolongada en el neonato con hipotermia que ponga en riesgo su salud, se deben aplicar medidas de control térmico que favorezcan una termorregulación eficaz desde el momento del nacimiento. Cada minuto que pasa es decisivo, por lo que el personal de enfermería debe identificar de manera temprana los riesgos en el neonato, valorar correctamente la temperatura en el sitio y dispositivos correctos para un monitoreo preciso y seguro que permita orientar sus intervenciones en el mínimo de tiempo ${ }^{19}$.

En relación con el tiempo ideal para lograr la eutermia en el neonato de riesgo, se considera que se debe iniciar el control térmico como una medida prioritaria debido a que, durante los primeros 10 a 20 minutos, el neonato pierde de manera fisiológica de 2 a $4{ }^{\circ} \mathrm{C}$ al ser expuesto bruscamente del medio intrauterino al medio ambiente, por lo que se debe propiciar el mantenimiento de la temperatura normal durante la primera hora de vida que es considerada como «la hora dorada» en el proceso de adaptación del neonato y donde surgen las complicaciones derivadas de la presencia de riesgos para la salud y la atención brindada por el equipo de salud ${ }^{2,20}$.

A pesar de ello, hay estudios que muestran que es difícil lograr la eutermia en los neonatos de riesgo en el mínimo de tiempo, como lo es la «hora dorada», por lo que, de acuerdo a las pocas investigaciones encontradas, se determina que el tiempo en que se debe termorregular a un recién nacido en el ámbito hospitalario debe ser en un máximo de 6 horas, dependiendo de las cifras de la temperatura valorada desde el nacimiento, ya que hay autores que coinciden en que se debe medir la temperatura del recién nacido cada 15 a 30 minutos, desde su nacimiento, durante el trasporte y en las primeras horas de vida durante su hospitalización en la terapia intensiva neonatal, hasta lograr la eutermia, ya que, según la literatura consultada, la temperatura corporal del neonato debe subir $0.5^{\circ} \mathrm{C}$ cada hora hasta lograr la eutermia ${ }^{18-21}$, y es el personal de enfermería el principal responsable de su cuidado, de acuerdo a lo expuesto por Florence Nigthingale, quien señaló conservar la energía vital del paciente para que la naturaleza actúe sobre él ${ }^{22}$, en este caso la temperatura ambiental, por lo que es necesario partir de colocar al neonato en las mejores condiciones posibles, centradas en el medio ambiente y creando un entorno térmico saludable, necesario para aplicar cuidados de enfermería seguros y de calidad para disminuir con ello las complicaciones derivadas de la presencia de hipotermia, motivo por el cual se planteó la presente investigación.

\section{Objetivo}

Determinar la asociación entre factores de riesgo de hipotermia con el tiempo de termorregulación eficaz 
del neonato hospitalizado en una unidad de cuidados intensivos neonatales en Tamaulipas, México.

\section{Material y métodos}

Estudio realizado mediante diseño cuantitativo, de tipo retrospectivo, descriptivo correlacional en 155 neonatos de ambos sexos, con edad gestacional y peso indistinto al nacer, que cursaron con hipotermia a su ingreso a la UCIN durante el periodo comprendido entre agosto de 2017 y julio de 2018. Se excluyeron los neonatos diagnosticados con encefalopatía hipóxica-isquémica. La población de estudio se determinó mediante muestreo probabilístico aleatorio simple. La recolección de los datos se realizó mediante la revisión documental de los expedientes clínicos de los neonatos hospitalizados, considerando las variables de interés para la investigación, las cuales se registraron en formato diseñado ex profeso por las autoras, el cual fue denominado «Presencia de factores de riesgo de hipotermia en el neonato hospitalizado", y validado mediante la opinión de 5 expertos en neonatología, y se obtuvo un alfa de crombach de 0.75 Dicho instrumento contenía datos sociodemográficos para caracterizar a la población de estudio, como son edad gestacional, sexo, peso, tipo de nacimiento, grado de hipotermia presentada, así como presencia de factores de riesgo para hipotermia, en los cuales se engloban Apgar al nacimiento, necesidad de reanimación neonatal, antecedentes de hipoglicemia, presencia de anomalías congénitas, presencia de RCIU, entre otras. Del mismo modo, se registraron factores de riesgo relacionados con el cuidado del neonato, como uso de incubadora, colocación del neonato cerca de corrientes de aire, uso de ropa precalentada, aplicación de baño temprano, entre otros. Asimismo, se registró la temperatura al ingreso, el tiempo en que se logró una termorregulación eficaz y temperatura alcanzada. Para todo ello, se utilizó el cuestionario con respuesta cerrada de tipo dicotómica (Sí/NO), realizado previa autorización por el Comité de Ética e Investigación del Hospital con registro 117/2018/SCEI. Los datos obtenidos fueron capturados en el Programa IBM Statistical Package for the Social Sciences (SPSS V.23). El análisis de las variables sociodemográficas para caracterizar a los neonatos estudiados se realizó mediante estadística descriptiva a través de distribución de frecuencias y porcentajes. La asociación entre las variables de estudio (factor de riesgo y tiempo de hipotermia) se determinó mediante análisis bivariado, para lo cual se utilizó estadística inferencial mediante la prueba de distribución de riesgo relativo, denominada odds ratio y Ji cuadrada de Pearson.

\section{Discusión de los resultados}

Al término de la investigación se observó que, de los 155 neonatos estudiados, presentaron hipotermia leve $13(8.4 \%)$ e hipotermia moderada 142 (91.6\%), ninguno presentó hipotermia grave a su ingreso a la UCIN. Esto es similar a lo reportado en la literatura, donde autores mencionan que hay una alta incidencia de hipotermia moderada a grave en el $93 \%$ de los neonatos que son hospitalizados en las $\mathrm{UCIN}^{2,4,10}$.

El tiempo de hipotermia que trascurrió hasta lograr la eutermia en los neonatos estudiados fue en promedio de 14 horas, con un mínimo de 1 hora y un máximo de 84 horas, DE 13.7 en un rango de 84 horas. De ellos, 134 (86.4\%) lograron la eutermia durante las primeras 24 horas: 59 (38\%) durante las primeras 6 horas, $30(19.35 \%)$ antes de las 12 horas y 45 (29.03\%) antes de las 24 horas. El resto (21 [13.5\%]) alcanzó la eutermia posterior a las 24 horas. Estos resultados son contrarios a lo presentado en neonatos hospitalizados en una unidad de cuidados intensivos en la India, donde se observó que en el $100 \%$ de casos lograron eutermia durante la primera hora de vida ${ }^{2}$, lo que es relevante analizar, ya que, según la literatura, la eutermia se debe procurar en las primeras 6 horas de nacimiento. Hay que considerar también que el sobrecalentamiento rápido ocasiona incremento del metabolismo cerebral, entre otros problemas que ponen en riesgo la salud del neonato con hipotermia (Fig. 1).

En nuestro estudio encontramos que el tipo de hipotermia se asocia al tiempo que trascurre en el neonato hasta alcanzar la eutermia ( 6 horas [OR: 1.891; IC 95\%: 1.240-2.883], > 6 horas [OR: 0.485; IC 95\%: 0.2131-108]). A este respecto no encontramos algún estudio relacionado para discutir nuestros resultados, a pesar de ello, los resultados ponen de manifiesto la necesidad de que el personal de enfermería valore el tipo de hipotermia que presenta el recién nacido, ya que, según nuestros resultados, a mayor grado de hipotermia, mayor tiempo en alcanzar la normotermia. Ello hace énfasis en la importancia de la valoración de riesgo, tipo de hipotermia y tiempo, considerando lo reportado en algunos estudios, donde mencionan que durante el nacimiento se inicia el proceso de adaptación extrauterina, y la temperatura es uno de las funciones que se ven afectadas en el neonato de manera temprana ${ }^{2,6,7}$ al perder de manera fisiológica de 2 a $4{ }^{\circ} \mathrm{C}$ de temperatura durante los primeros 20 minutos posteriores al 


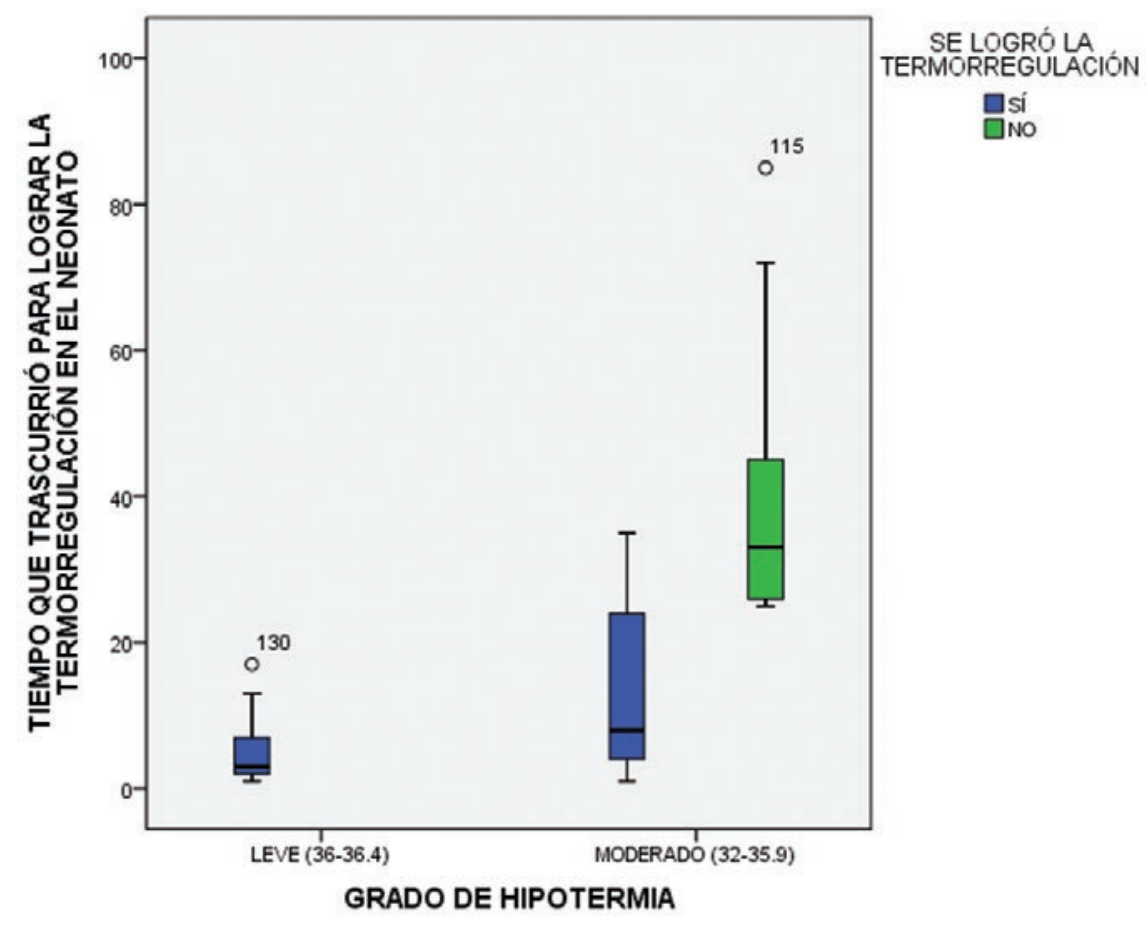

Figura 1. Termorregulación eficaz según el grado de hipotermia en neonatos hospitalizados ( $\mathrm{n}=155$ ). Fuente: instrumento de recolección de datos (IRD).

nacimiento. Al contrario de lo que ocurre en los neonatos con encefalopatía hipóxico-isquemica, en que el neonato debe permanecer en hipotermia durante las primeras horas de vida como medida terapéutica de protección cerebral, en los neonatos de riesgo existe la necesidad de lograr la eutermia durante las primeras horas de vida para evitar posibles complicaciones ${ }^{17}$.

Dentro de las características personales de los recién nacidos con presencia de hipotermia, 80 (51.6\%) son del sexo masculino, 72 (46.5\%), de sexo femenino y $3(1.9 \%)$, con sexo indiferenciado. Al analizar el sexo como factor de riesgo para la presencia de hipotermia no se encontró asociación entre dichas variables $\left(X^{2}=1.445 ; p>0.05\right)$. Son resultados similares a lo reportado en la literatura, donde se menciona que tienen el mismo riesgo de hipotermia niños y niñas, siempre y cuando no exista otro factor asociado, al contrario de algunas patologías específicas, en que el sexo es predominante para la presencia de enfermedad $2,3,10,11$

En cuanto a su clasificación de edad por semanas de gestación, según la valoración de Capurro, la mayoría de los recién nacidos (76 [49\%]) eran a término, seguidos de neonatos pretérmino (72 [46.5\%]), inmaduros $(6[3.9 \%])$ y postérmino $(1[0.6 \%])$, y se encontró relación entre las variables $\left(X^{2}=591.71 ; p<0.05\right)$. La edad gestacional media fue de 36.3 semanas de gestación, con una edad mínima de 26 semanas de gestación y una edad máxima de 45 semanas de gestación, DE +3.25 semanas de gestación. Esto es lo contrario a lo reportado en investigaciones realizadas en distintos lugares a nivel mundial ${ }^{3-5}$, donde se ha encontrado que son los neonatos pretérmino los que mayormente presentan hipotermia desde el nacimiento, por lo que son necesarias las medidas para prevenir las pérdidas de calor para mantener un ambiente térmico neutro, disminuir el consumo de oxigeno y el estrés metabólico.

El promedio del peso de los neonatos con hipotermia fue de 2,475 g, con un mínimo de $500 \mathrm{~g}$ y un máximo de 4,690 g, DE +925.41 g, sin relación entre peso y presencia de hipotermia $\left(X^{2}=122.46 ; p>0.05\right)$. Esto es lo contrario a lo reportado en la literatura, donde se menciona que, a menor peso, como es el caso de los neonatos pretérmino o con RCIU, mayor presencia de hipotermia ${ }^{2-4,10}$. Nuestros resultados coinciden con lo reportado por Ruschel, et al., quienes expusieron que los neonatos a término también sufren de hipotermia, especialmente cuando son expuestos sin protección al 


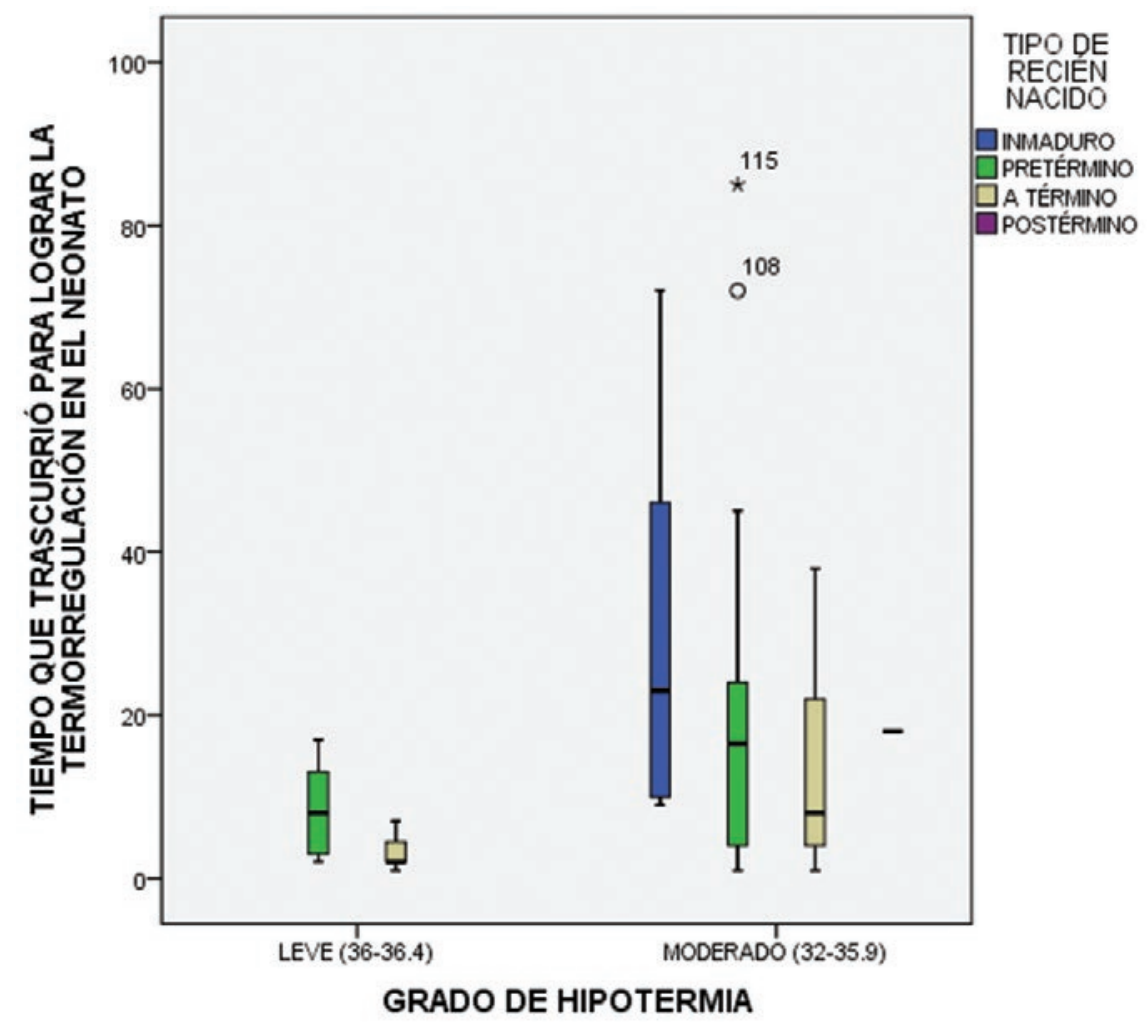

Figura 2. Asociación entre el grado de hipotermia y el tiempo de termorregulación de acuerdo al tipo de neonato ( $\mathrm{n}=155)$. Fuente: instrumento de recolección de datos (IRD).

medio ambiente frío, no se procura el contacto piel a piel, ni el apego inmediato al nacer y, además, son bañados de manera temprana durante su estancia hospitalaria $^{14}$ (Fig. 2).

Entre los factores de riesgo que se presentaron en los neonatos con hipotermia, se encuentran la presencia de anomalías congénitas en 25 (16.1\%), que se asociaron a hipotermia (leve [OR: 0.941; IC 95\%: 0.223-4.00], moderada [OR: 1.005; IC 95\%: 0.885 1.141]. En nuestro estudio encontramos que los neonatos con algún defecto congénito, especialmente con anomalías de la pared abdominal, tienen un riesgo mayor de lograr la eutermia posterior al tiempo establecido (< 6 horas [OR: 0.674; IC: $0.348-1.305]$ ], > 6 horas [OR: 1.232; IC 95\%: 0.927-1.636]). Son resultados similares a lo encontrado en la literatura, donde se menciona que los neonatos con alguna anomalía congénita son más propensos de perder calor por evaporación y conducción $n^{12,14,19}$.

El RCIU, considerado como riesgo para hipotermia, se presentó en 49 (31.6\%) casos (leve [OR: 0.393; IC
95\%: 0.091-1.702], moderada [OR: 1.070; IC 95\%: 0.981-1.167]), lo que se asoció a un mayor tiempo en alcanzar la eutermia neonatal (< 6 horas [OR: 0.424 ; IC 95\%: 0.236-0.763], > 6 horas [OR: 1.534; IC 95\%: 1.217-1.934]). Ello es similar a lo descrito en investigaciones previas $2,10,12$, que mencionan que el $54 \%$ de los neonatos con RCIU sufren de hipotermia moderada a leve durante sus primeras horas de vida, especialmente cuando se realizan prácticas inadecuadas de control, al ser valorado como a término y ser expuesto al medio ambiente frío, sin procurar el contacto piel a piel, el apego inmediato y ser bañados de manera temprana durante su estancia hospitalaria ${ }^{13,14}$.

Fueron obtenidos por cesárea 118 (76.1\%), por parto fisiológico 25 (16.1\%) y distócico 12 (7.7\%). El tipo de nacimiento, según algunos autores, es considerado como de riesgo. En nuestro estudio se identificó asociación de dicha variable para la hipotermia (leve [0.902; IC 05\%: 0.212-2.832], moderada [OR: 1.009; IC 05\%: 0.892-1.141]), lo que repercutió en el tiempo de hipotermia neonatal $(<6$ horas [OR: 1.215; IC 95\%: 


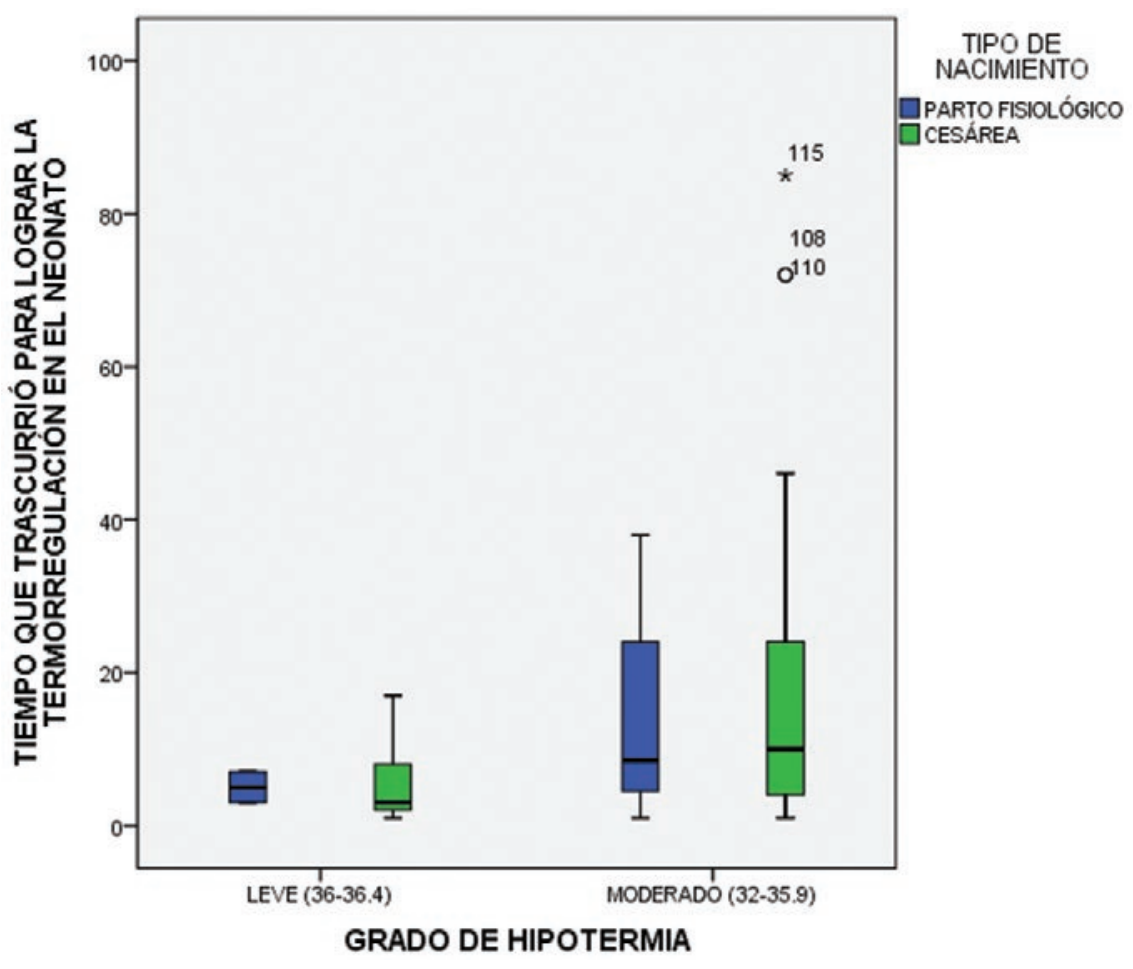

Figura 3. Asociación entre el grado de hipotermia y el tiempo de termorregulación según el tipo de nacimiento ( $\mathrm{n}=155)$.

Fuente: instrumento de recolección de datos (IRD).

0.759-1.944], > 6 horas [OR: 0.868; IC 95\%: 0.593-1.270]). Estos datos son similares a lo reportado por algunos autores, quienes mencionan que los nacidos por cesárea incrementan el riesgo 2:1 de presentar hipotermia, ello se relaciona con la sedación, el tiempo de intervención, la falta de contacto piel a piel y la exposición a temperatura menor a $23^{\circ} \mathrm{C}$ en el área quirúrgica, lo que propicia un mayor tiempo de hipotermia y dificultad para lograr la termorregulación de manera eficaz ${ }^{11-13}$ (Fig. 3).

Los neonatos estudiados se evaluaron con un Apgar al nacer de $8 / 8$ en la mayoría de los casos, $75(48.4 \%)$, de $9 / 945$ (29\%) y de $7 / 835$ (22.6\%). Al este respecto se reportó que requirieron reanimación con puntas nasales $58(37.4 \%)$ de los casos, $34(21.9 \%)$ con presión positiva y $63(40.6 \%)$ no requirió intervención especial al nacimiento. Este factor de riesgo se relacionó con hipotermia (leve [OR: 1.524; IC 95\%: 0.49-4. ${ }^{6}$, moderada [OR: 0.958; IC 95\%: 0.841-1.090]). De los 155 neonatos estudiados, 35 (22.6\%) tuvieron antecedente de hipoxia leve, lo que se asoció a mayor tiempo en alcanzar la termorregulación ( $<6$ horas [OR: 0.754; IC 95\%: 0.443-1.286], > 6 horas [OR 1.176; IC 95\%: 0.8971.541]), tal como se menciona en la literatura, que hace referencia a que uno de los factores asociados a hipotermia es la presencia de hipoxia, problema que propicia el incremento de necesidades calóricas ante la disminución de oxígeno y generación de estrés metabólico ${ }^{2,6,16}$.

Presentaron hipoglucemia 57 (36.8\%) casos. En la literatura, esta variable se reporta como factor de riesgo para hipotermia, considerando que la mayoría de los neonatos con hipoglucemia disminuye su metabolismo basal, lo que resultado en una disminución de energía calórica $^{15,16}$. En nuestro estudio, de forma similar, la hipoglucemia se asoció a hipotermia moderada (leve [OR: 0.313; IC 95\%:0.72-1.31], moderada [OR: 1.087; IC 95\%: 0.997-1.185]). En relación con el tiempo de hipotermia se encontró que los neonatos con hipoglucemia tardaron más de 6 horas en lograr termorregulación (< 6 horas [OR: 0.611; IC 95\%: 0.386-0.976], > 6 horas [OR: 1.330; IC 95\%: 1.042-1.698]).

En relación con los factores de riesgo asociados al cuidado de enfermería, encontramos que los neonatos que tuvieron contacto con objetos no precalentados fueron 95 (61.3\%), ello se asoció a hipotermia (leve [OR: 1.421; IC 95\%: 0.458-4.410], moderada [OR: 0.971; IC 


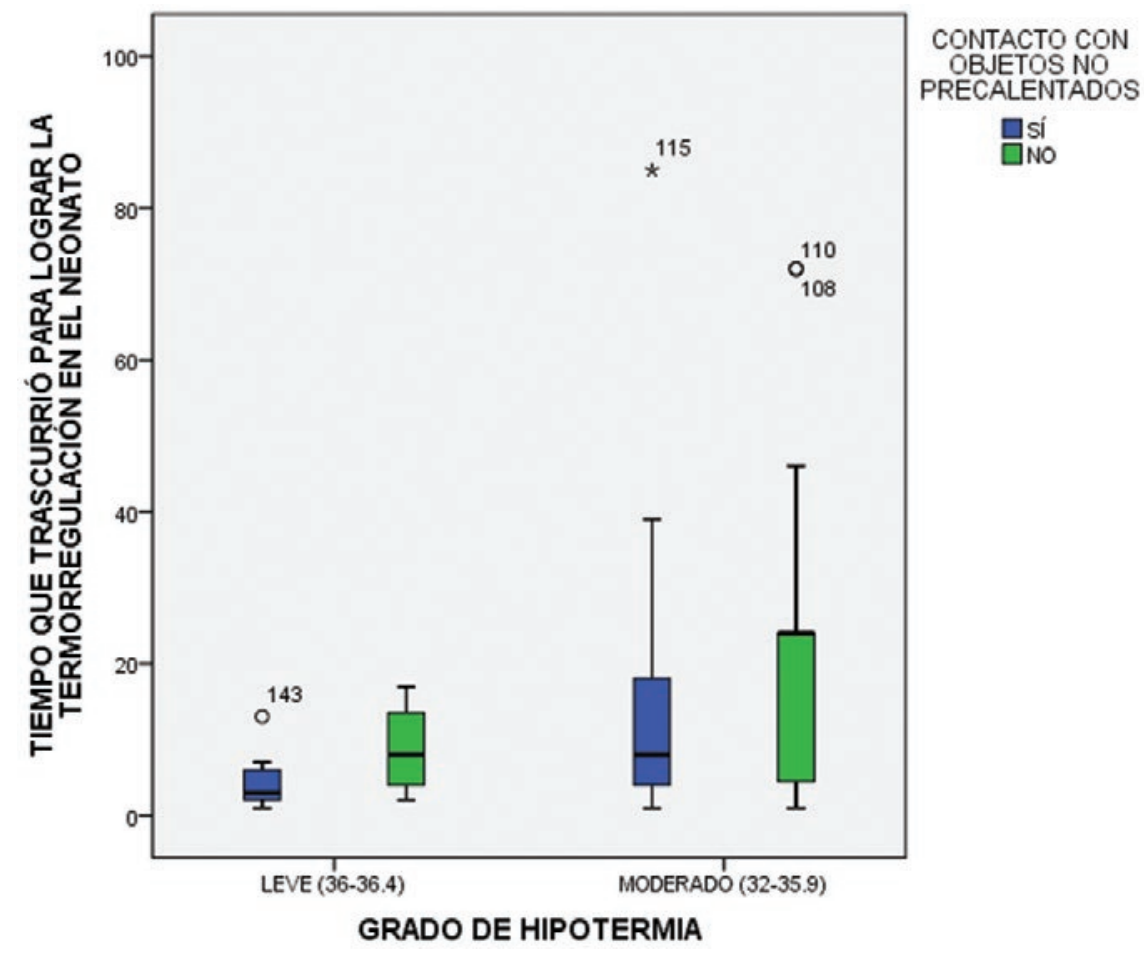

Figura 4. Asociación entre el grado de hipotermia y el tiempo de termorregulacion según el registro de contacto con objetos no precalentados $(\mathrm{n}=155)$.

Fuente: instrumento de recolección de datos (IRD).

95\% 0.883-1.065]), lo que repercutió en el tiempo en alcanzar la eutermia al perder calor por conducción $(<6$ horas [OR: 1.396; IC 95\%: 0.904-2.157], > 6 horas [OR: 0.816; IC 95\%: 0.637-1.047]). Son resultados similares a lo reportado en la literatura, donde se observa que neonatos a los cuales se les dio atención con ropa no precalentada, en cunas e incubadoras no reguladas de manera correcta a $34{ }^{\circ} \mathrm{C}$ sufrieron hipotermia desde el momento del nacimiento $4,9,10,15$ (Fig. 4).

Tuvieron contacto con circulación de aire frío 90 $(58.1 \%)$. Este factor se considera de riesgo para la hipotermia, como se observó en nuestro estudio (leve [OR: 1.156; IC 95\%: 0.396-3.375], moderada [OR 0.987; IC 95\%: 0.897-1.086]). A pesar de ello, los neonatos lograron la eutermia en el tiempo establecido para ello, disminuyendo, así, el riesgo de pérdidas de calor por convección (< 6 horas [OR: 1.280; IC 95\%: 0.846-1.937], $>6$ horas [OR: 0.857; IC 95\%: 0.667-1.697]). Son resultados similares a lo encontrado en la literatura ${ }^{4,16}$, donde se reporta que existe una alta incidencia de hipotermia en neonatos que son atendidos en unidades que se encuentran cerca de corrientes de aire frío, lo que interfiere con el tiempo de hipotermia, debido al tiempo en que tardan las intervenciones del equipo de salud en la atención del neonato (Fig. 5). En relación con el tiempo en que se logró la termorregulación eficaz, nuestros resultados coinciden con Aliona, et al., que mencionan que al estar preparados con el entorno y equipamiento correcto para la atención del neonato desde el nacimiento hace que se propicie la termorregulación en 81 minutos aproximadamente posteriores al nacimiento ${ }^{13}$. Este dato es similar al mencionado por Bergstom, et al, quienes reportan eutermia alcanzada a los 90 minutos durante la hospitalización posterior al nacimiento ${ }^{15}$.

Fueron inmersos en agua para ser bañados $8(5.2 \%)$, lo cual se asoció a hipotermia (leve [OR: 1.536; IC 95\%: 0.226-10.36], moderada [OR: 0.953; IC 95\%: 0.730 1.244]). Se observó que el baño favoreció la presencia de hipotermia mayor a 6 horas ( $<6$ horas [OR: 0.623; IC 95\%: 0.185-2.102], > 6 horas [OR: 1.253; IC 95\%: $0.822-1.909])$. Al respecto se menciona que no es necesario realizar el baño todos los días, ya que el vérmix ayuda a regular la temperatura del neonato, por lo que se debe absorber solo por la piel. Al realizar el baño de manera temprana se disminuye el papel protector 


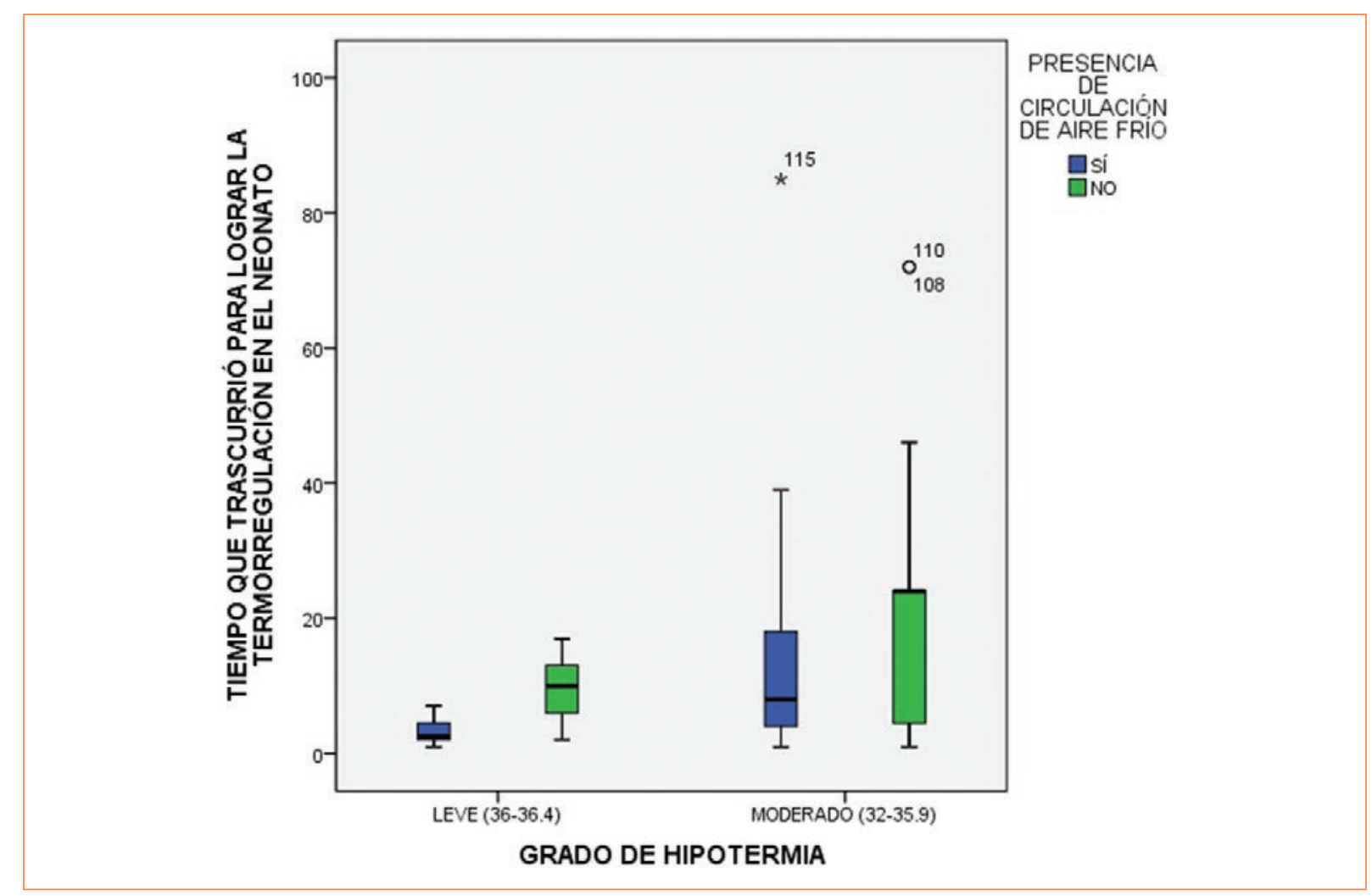

Figura 5. Asociación entre el grado de hipotermia y el tiempo de termorregulación en neonatos con registro de presencia de circulación de aire frío $(n=155)$.

Fuente: instrumento de recolección de datos (IRD).

del vérmix, por lo que se recomienda posterior a las 24 horas $^{9}$. Ello lo sugieren algunos autores que encontraron que neonatos que fueron bañados antes de las 6 horas presentaron hipotermia de moderada a grave en un $93 \%$ de los $\operatorname{casos}^{4,13}$. En nuestro estudio, solo a un pequeño porcentaje de neonatos se les dio baño de manera temprana en la UCIN.

Fueron manejados en cuna térmica $100(64.5 \%)$ casos y en incubadora 55 (35.5\%), dependiendo del diagnóstico de ingreso y tipo de neonato ${ }^{18,19}$ como sugiere la literatura para proveer de calor al neonato y facilitar su proceso de termorregulación. Es importante mencionar que en la mayoría de los casos (103 [66.5\%]), enfermería documenta en la hoja de registros clínicos el diagnóstico enfermero de hipotermia neonatal (62 [40.4\%]) y termorregulación ineficaz (41 [26.1\%]). El resto no considera estos diagnósticos en su valoración inicial, de acuerdo a lo sugerido en la literatura ${ }^{19}$, que permitan evidenciar el cuidado otorgado por el personal de enfermería como menciona Ninghtingale en su teoría ${ }^{20-22}$, con la finalidad de establecer un plan de cuidados acorde a la valoración de riesgos y las necesidades detectadas en el neonato, especialmente en el tiempo en que se debe lograr la adaptación fisiológica al medio extrauterino, y que ello repercuta en el pronóstico de salud del neonato hospitalizado (Fig. 5).

\section{Conclusión}

En nuestro entorno encontramos que hay una incidencia de hipotermia neonatal del $4.4 \%$, en la que predomina la hipotermia moderada $\left(32-35.9^{\circ} \mathrm{C}\right)$ en el $91.6 \%$ de los casos. El tiempo de hipotermia en promedio fue de 14 horas, el $38 \%$ de los neonatos lograron eutermia durante las primeras 6 horas (OR: 1.891; IC 95\%: 1.240-2.883). Entre los factores de riesgo relacionados con el tiempo de hipotermia se encuentran la edad (a término el $49 \%\left[X^{2}=591.71 ; p<0.05\right]$; presencia de anomalías congénitas (16.1\% [OR 1.005; IC 95\%: 0.885-1.141], > 6 horas [OR: 1.232; IC 95\%: 0.9271.636]); retardo en el crecimiento intrauterino (31.6\% [OR 1.070; IC 95\%: 0.981-1.167], > 6 horas [OR 1.534; IC 95\%: 1.217-1.934]); obtenidos por cesárea (76.1\% [OR: 1.009; IC 05\%: 0.892-1.141], < 6 horas [OR: 1.215; IC 95\%: 0.759-1.944]); Apgar bajo al nacer (48.4\% [OR: 
1.524; IC 95\%: 0.49-4.6, > 6 horas OR 1.176; IC 95\%: $0.897-1.541)$; antecedente de hipoglucemia $(36.8 \%$, [OR: 1.087; IC 95\%: 0.997-1.185], > 6 horas [OR: 1.330; IC 95\%: 1.042-1.698]); tener contacto con objetos no precalentados (61.3\% [OR: 1.421; IC 95\%: 0.458$4.410],<6$ horas [OR: 1.396; IC 95\%: 0.904-2.157]); tener contacto con circulación de aire frío $(58.1 \%$, [OR: 1.156; IC 95\%: 0.396-3.375], < 6 horas [OR: 1.280; IC 95\%: 0.846-1.937]); e inmersos en agua para ser bañados (5.2\% [OR: 1.536; IC 95\%: 0.226-10.36], > 6 horas [OR: 1.253; IC 95\%: 0.822-1.909]). Todo lo anterior pone de manifiesto la importancia de la valoración temprana de los factores de riesgo con la finalidad de establecer un plan de cuidados seguro y de calidad para ser implementado en el tiempo ideal por el equipo de salud liderado por enfermería, y, con ello, disminuir la presencia de complicaciones derivadas de la hipotermia en el neonato.

\section{Agradecimientos}

A Cuellar Jáuregui Maricruz, Hernández de la Torre Eunice, Lores Ortega Carmen Lorena y Urbina González Luz Mayerling por su colaboración en la captura de datos.

\section{Conflicto de intereses}

Los autores declaran que no existe conflicto de intereses.

\section{Responsabilidades éticas}

Protección de personas y animales. Los autores declaran que para esta investigación no se han realizado experimentos en seres humanos ni en animales.

Confidencialidad de los datos. Los autores declaran que han seguido los protocolos de su centro de trabajo sobre la publicación de datos de pacientes.

Derecho a la privacidad y consentimiento informado. Los autores declaran que en este artículo no aparecen datos de pacientes.

\section{Bibliografía}

1. North American Nursing Diagnosis Association. Diagnósticos enfermeros. Definiciones y clasificación 2015-2017. Ed. Elsevier.

2. Shaw SC, Devgan A, Anila S, Anushree N, Debnath H. Use of Plan-DoStudy-Act cycles to decrease incidence of neonatal hypothermia in the labor room. Med J Armed Forces India. 2018;74(2):126-32.

3. García Muñoz R, Rivero Rodríguez S, Siles Quezada C. Factores de riesgo de hipotermia al ingreso en recién nacidos de muy bajo peso y morbimortalidad asociada. Rev Annals Pediatric. 2014;80(3):135-202.

4. Luke C. Mullany. Neonatal hypothermia in low-resource settings. Seminars in Perinatology. 2010;34(6):426-33.

5. Zamorano Jiménez CA, Cordero González G,mFlores Ortega J, Baptista González HA, Fernández Carrocera LA. Control térmico en el recién nacido pretérmino. Perinatología y Reproducción Humana. 2012;26(1): 43-50.

6. Sociedad Iberoamericana de Neonatología. Guía de práctica clínica de termorregulación en el recién nacido. Argentina, 2010.

7. De Gracia Dionicio, Christian José. Factores de riesgo asociados a hipotermia neonatal en la Unidad de Neonatología del Hospital Regional Docente de Trujillo. Tesis de Medicina. Universidad Nacional de Trujillo; 2018.

8. Secretaria de Salud. Norma Oficial Mexicana NOM 007 SSA2 2016. Para la atención de la mujer durante el embarazo, parto y puerperio y de la persona recién nacida.

9. Instituto Mexicano del Seguro Social. Guía de práctica clínica: Cuidados del recién nacido prematuro sano hospitalizado; 2018.

10. Wilson E, Maier RF, Norman M, Misselwitz B, Howell EA, Zeitlin J, et al. Admission hypothermia in very preterm infants and neonatal mortality and mobility. J Pediatric. 2016;175:61-7.

11. Baker B. Maternal Hypothermia in Scheduled Cesarean Births and Neonatal Outcomes. J Obstet Gynecol Neonatal Nurs. 2012.;41:S134-5.

12. Jensen C, Ebbesen F, Petersen J, Sellmer A, Bach C, Hendrickson T. Hypothermia at neonatal intensive care unit admission was not associated with respiratory disease or death in very preterm infants. Acta Paediatr. 2017; 106:1934-9.

13. Aliona Vilinsky BS, Sheridan A, Nugent LE. Preventing peri-operative maternal and neonatal hypothermia after skin-to-skin contact. J Neonat Nurs. 2016;22(4):163-70.

14. Ruschel LM, Pedrini DB, Cunha ML. Hypothermia and the newborn's bath in the first hours of life. Revista Gaucha de Enfermagem. 2018; $39,:$ e20170263

15. Bergström A, Byaruhanga R, Okong P. The impact of newborn bathing on the prevalence of neonatal hypothermia in Uganda: A randomized, controlled trial. Acta Pædiatrica. 2005;94:1462-7.

16. De Siqueiros Caldas JP, Castro Miller F, Fernandez de Camargo J. Cavalcautti Castro PA. Effectives of a measure program to prevent admission hypothermia in very low-birth weight preterm infants. J Ped. 2018;9(4):368-73.

17. Grupo de Trabajo sobre Hipotermia Terapéutica, Sociedad Argentina de Pediatría. Recomendaciones para el tratamiento con hipotermia en el recién nacido con encefalopatía hipóxico isquémica. Arch Argent Pediatr. 2017;115 supl 3:s38-s52.

18. Alcocer Arreguin C, Sauviñon Tejeda P, Gil Rosales C, Calderón Jiménez C. Hipotermia corporal inducida en recién nacidos con asfixia. Rev Mex Pediat. 2013;80(5):179-84.

19. Kristine A. Karlsen. The S.T.A.B.L.E. Program. Cuidados Post-reanimación y Pre-Transporte para Neonatos Enfermos. Guía para Personal de Salud Neonatal. 5. ${ }^{\text {a }}$ ed. March of Dimes, 2006. p. 43-61.

20. Chang HY, Sung YH, Wang SM, Lung HL, Chang JH, Hsu CH. Short and long term outcomes in very low birth weight infants with admission hypothermia. PLoS One. 2015;10(7):e0131976.

21. Hsu KH, Chiang MC, Lin JJ, Wang YC, Lie R. Thermal blanket to improve thermoregulation in preterm infant. Pediatr Crit Care Med. 2015; 16(7):637-43.

22. Rioja Rendón KK, Cigarro Carre NG, Zarate Grajales RA. Florence Ninghtingale: principios y evolución en la gestión de calidad del siglo XX. Rev Mex Enfer. 2015;3(1):13-6. 